\title{
How to design clinical rehabilitation trials for the upper paretic limb early post stroke?
}

\author{
Caroline Winters ${ }^{1,2}$, Martijn W. Heymans ${ }^{3,4}$, Erwin E. H. van Wegen ${ }^{1,2}$ and Gert Kwakkel ${ }^{1,2,5,6^{*}}$
}

\begin{abstract}
Background: The impact of spontaneous neurobiological recovery is still neglected in designing rehabilitation trials early post stroke. We aimed to investigate the impact of the timing of randomization and prognostic stratification on the required sample sizes that are needed to reveal significant intervention effects on upper limb function at 26 weeks after first-ever ischemic stroke.
\end{abstract}

Method: Sample size calculations were based on a cohort study of 159 patients, using the Fugl-Meyer Assessment Upper Extremity and Action Research Arm Test as outcome measures (power $=80 \%$; two-tailed alpha $=0.05$ ). We investigated different scenarios: random sampling of patients within five time intervals (stroke onset to 1, 3, 5, 8 and 12 weeks post stroke), and within stratified groups according to the presence or absence of voluntary extension of the thumb and/or two or more fingers at intake.

Results: The heterogeneity between outcome scores of patients, and subsequently the required sample sizes, increased from the first to the fifth time interval. Compared to the whole group, the sample sizes for both stratified groups (i.e., patients with and without Voluntary Finger Extension (VFE)) were lower. The required sample sizes for the patient group without VFE markedly increased when the time interval was broadened from 1 to 12 weeks post stroke, as opposed to the decrease seen for the group of patients with VFE.

Conclusion: These results are fundamental for designing upper limb trials early post stroke. To prevent type II error, future upper limb trials should randomize patients at a fixed moment early post stroke and stratify patients according to their potential neurobiological recovery.

Trial registration: Netherlands Trial Registry, www.trialregister.nl, NTR1424, registered on 27 August 2008.

Keywords: Stroke, Randomized controlled trial, Power, Sample size, Prognostic stratification, Upper extremity

\section{Background}

Recent systematic reviews and meta-analyses of stroke rehabilitation trials that are started early after stroke show that the effect sizes of interventions are small to moderate and account for 5 to $10 \%$ of the differences in outcome [1, 2]. Approximately $98 \%$ of all randomized controlled trials (RCTs) are proof-of-concept trials and are often heavily underpowered [1]. At this moment,

\footnotetext{
* Correspondence: g.kwakkel@vumc.nl

'Department of Rehabilitation Medicine, VU University Medical Center, MOVE

Research Institute Amsterdam, Amsterdam, The Netherlands

${ }^{2}$ Neuroscience Campus Amsterdam, Vrije Universiteit, Amsterdam, The

Netherlands

Full list of author information is available at the end of the article
}

there is no evidence that stroke rehabilitation programs started within the first 3 months post stroke are more effective than programs initiated beyond this time period, despite the growing evidence of heightened brain plasticity early post stroke [3-5].

Only about $7 \%(N=18)$ of the clinical stroke trials that focus on upper limb recovery performed the randomization procedure within the first 2 weeks after stroke onset (i.e., hospital-based trials) [1]. The majority of RCTs start their randomization procedure when patients are discharged from the hospital and admitted to a rehabilitation ward or nursing home [1]. As a consequence, inclusion of subjects in most phase II trials ranges from a few days up to several months post stroke. Such pragmatic design of RCTs ignores 
the impact of spontaneous neurobiological recovery during the first 5 to 10 weeks post stroke which accounts for about $80 \%$ of all neurological improvement that is clinically observed in longitudinal cohort studies of the upper limb, lower limb and cognitive impairments [6-9]. One may, therefore, raise the fundamental question whether the timing of randomization in the first 12 weeks post stroke is an important factor for designing phase II trials in stroke rehabilitation. One may hypothesize that the arbitrary timing of randomization post stroke causes type II errors in small RCTs through the additional variance that is introduced by still poorly understood, time-dependent processes of spontaneous neurobiological recovery early post stroke.

Furthermore, several systematic reviews and metaanalyses suggest that evidence-based therapies for the upper paretic limb are strongly dependent on an appropriate selection of patients at baseline $[10,11]$. Several prospective cohort studies showed that the ability to voluntarily extend one or more fingers against gravity within the first 3 days is a robust clinical marker for upper limb recovery after 3 or 6 months post stroke [12,13], reflecting the intactness of the corticospinal tract [14]. Unfortunately, only one clinical trial out of the 266 upper limb trials published [1] stratified patients on the basis of their initial impairment prior to the randomization procedure [15]. At this moment, evidence-based interventions seem to be restricted to those patients with some Voluntary Finger Extension (VFE) [1, 16, 17]. One may hypothesize that the choice of whether or not to stratify patients prior to randomization based on early prognosis, for example using VFE, influences the heterogeneity in upper limb function between patients and consequently the probability of finding differential effects post intervention $[6,10,18]$.

The aims of the present study were to investigate the impact of (1) different time intervals that vary in length between stroke onset and randomization and (2) prognostic stratification based on the presence or absence of VFE, on the required sample size needed to reveal significant and clinically important intervention effects on the Action Research Arm Test (ARAT) and Upper Extremity motor section of the Fugl-Meyer Assessment (FMA-UE) at 26 weeks after stroke.

\section{Methods}

\section{Study population and procedure}

Data from the EXPLCIT-stroke trial were used [16]. Details of this RCT can be found elsewhere [16, 19]. The inclusion criteria were: (1) first-ever middle cerebral aftery ischemic stroke, (2) upper limb paresis according to item 5 of the National Institutes of Health Stroke Scale (NIHSS $>1$ point), (3) Mini Mental State Examination score $\geq 23$ points, (4) age between 18 and 80 years, (5) no upper limb musculoskeletal impairments, (6) no botulinum toxin treatment in the previous 3 months, (7) the ability to sit independently for $30 \mathrm{~s}$ and (8) provision of written informed consent.

At intake within 2 weeks post stroke, patients were stratified to (1) a group of patients presenting with VFE $(N=58)$ and randomly assigned to either modified Constraint-induced Movement Therapy (mCIMT) or usual care or (2) a group of patients presenting without VFE $(N=101)$ and randomly assigned to electromyogram (EMG)-triggered Neuro-muscular Stimulation (EMGNMS) or usual care. The patients with VFE had the ability to voluntarily extend the thumb and/or two or more fingers of the affected hand (to $10^{\circ}$ or more). The functional assessments were repeated weekly up to 5 weeks after stroke and at 8-, 12- and 26-week follow-ups [16, 19].

\section{Outcome measurements}

In the present study we used the ARAT and FMA-UE as primary outcome measures. The ARAT is an upper limb capacity test which assesses the ability to grasp, move and release objects of various sizes, weights and shapes. It has 19 subquestions scored on a 4-point ordinal scale, adding up to a total score between 0 and 57 points (57 = normal capacity) $[20,21]$. The Minimal Clinically Important Difference (MCID) was set at 5.7 points, i.e., $10 \%$ of the range [22]. The FMA-UE assesses limb impairment in terms of synergistic motor control. It has 22 subquestions scored on a 3 -point ordinal scale, adding up to a total score between 0 and 66 points (66 = normal function) $[23,24]$. The MCID was set at 6.6 points $[25,26]$.

\section{Statistical analysis}

Approximately $8 \%$ of the 1272 assessments in the EXPLICIT-stroke trial were missing due to various reasons (recurrent stroke, sickness, etc.) [16]. We estimated these missing data points using individual curve fitting for subjects with two or more available assessments by estimating the ARAT and FMA-UE recovery curves using a linear mixed model (linear and quadratic component) that best described the individual recovery pattern, and that accounted for the repeated measures. The estimated data were merged with the original data to create a new complete dataset and checked by visual inspection. All further analyses were performed on this new (modelled) dataset. A total of 157 out of the 159 patients were eligible for further analysis: one patient had only one available assessment and another patient's recovery was negatively influenced by open heart surgery 4 months post stroke.

For the first aim we randomly varied the length of the time interval from stroke onset to randomization for each patient. Five different time intervals were evaluated: stroke onset $\left(\mathrm{T}_{0}\right)$ to 1 week post stroke, $\mathrm{T}_{0}$ to 3 weeks, 
$\mathrm{T}_{0}$ to 5 weeks, $\mathrm{T}_{0}$ to 8 weeks and $\mathrm{T}_{0}$ to 12 weeks. Within each time interval patients $(N=157)$ were randomly selected, resulting in a dataset in which some patients were included with a follow-up measurement at 1 week, others at 2, 3, 4 or 5 weeks when the time interval of 5 weeks after stroke onset was used. In this way heterogeneity in recruitment period post stroke onset was guaranteed.

For the second aim we adopted the EXPLICIT-stroke trial patient allocation to either the group of patients with or without VFE at intake. Fifty-seven patients with VFE at intake were available for analysis (with one dropout as described above). To obtain equal groups, we randomly selected 57 out of the 100 patients without VFE at intake using random sampling in SPSS (version 22), taking into account the distribution of randomization.

The minimum number of subjects in each group that is needed to find a differential effect at 26-week follow-up was calculated using Eq. 1. This number per group was multiplied by 2 to obtain the total number of subjects where after $10 \%$ was added to account for dropouts. We used a standard $t$ test sample size calculation to assess group differences at 26-week follow-up, assuming a normally distributed outcome. The power was set at $80 \%$ and two-tailed alpha at 0.05 . The standard deviation (SD) was determined using randomly selected patients for each different post stroke time interval as explained above. Different scenarios were used, selecting: (1) all patients $(N=157),(2)$ a subgroup of 114 patients including 57 patients with VFE and 57 patients without VFE, (3) patients with VFE at intake $(N=57)$ and (4) patients without VFE at intake $(N=57)$, within the five different time intervals. The average of the SD of the two groups (Eq. 3) was used to calculate the Cohen's $d$ effect size (Eq. 2).

$$
\begin{aligned}
& N_{\text {group }}=2 \times \frac{\left[Z_{\alpha}+Z_{\beta}\right]^{2}}{d^{2}} \\
& d=\frac{\bar{x}_{1}-\bar{x}_{2}}{S D_{\text {pooled }}} \\
& S D_{\text {pooled }}=\frac{\sqrt{S D_{1}^{2}+S D_{0}^{2}}}{2}
\end{aligned}
$$

Where $N_{\text {group }}$ is the number of subjects per group; $Z_{\beta}=0.842 ; \quad Z_{\alpha}=1.96 ; d=$ Cohen's effect size; $\bar{x}_{1}-\bar{x}_{2}=$ group mean difference at 26 -week follow-up. This difference was set at the MCID of the ARAT and FMA-UE, respectively 5.7 and 6.6 points; $S D_{\text {pooled }}=$ the average of the standard deviation of the sample (full dataset, after individual curve fitting); and $S D_{1}$ and $S D_{0}=$ standard deviation for, respectively, the intervention and the control group (full dataset, after individual curve fitting).
The $S D_{\text {pooled }}$ values are presented as variances. Patients received an intervention after randomization. We therefore recalculated the sample size estimations by taking account of this intervention effect by deriving the $S D_{\text {pooled }}$ from a linear regression model that included the intervention group variable, with as outcome the FMA-UE and ARAT score. The (square root of the) unexplained variances from this model were used as the $S D_{\text {pooled }}$ "controlled for" the intervention effect. As these were the same as the raw $S D_{\text {pooled }}$ values, we will present sample size estimations using the raw $S D_{\text {pooled }}$ values. Analyses were performed using $\mathrm{R}$ (version 3.1.1), unless otherwise indicated.

\section{Results}

Table 1 shows the characteristics of the 157 patients included in the present study. The "individual curve fitting" method was found to be successful after visual inspection of the individual recovery curves on the FMA-UE and ARAT. The FMA-UE and ARAT recovery curves are presented in Fig. 1.

Changing the time interval between stroke onset and randomization showed an increase in the required sample size to obtain an effect beyond MCID from the first to fifth time intervals for 148 subjects for the FMA-UE and 228 subjects for the ARAT (Table 2 and Fig. 2). The largest increase was visible between the first two time intervals for both outcome measures $\left(\triangle \mathrm{FMA}-\mathrm{UE}_{\mathrm{T} 1-\mathrm{T} 2}=77\right.$ subjects and $\triangle \mathrm{ARAT}_{\mathrm{T} 1-\mathrm{T} 2}=110$ subjects).

When patients were not stratified based on VFE, the required sample size for the first time interval (i.e., $\mathrm{T}_{0}$ to 1 week post stroke) was 308 and 218 subjects for, respectively, the FMA-UE and ARAT (Table 3 and Fig. 3). In comparison to the whole group $(N=114)$, the required sample sizes for the group of patients without VFE were lower; respectively, 44 and 9 subjects for the FMA-UE and ARAT. For the group of patients with VFE, the required sample size for the FMA-UE was also lower in comparison to the whole group (211 subjects). The required sample size with the ARAT as outcome measure was slightly higher due to a greater heterogeneity between patients (235 subjects; see also the individual recovery patterns in Fig. 1).

Table 1 Patient characteristics

\begin{tabular}{ll}
\hline Determinants & $N=157$ \\
\hline Sex, male/female ( $N)$ & $94 / 63$ \\
Age, years (mean \pm SD) & $59.9 \pm 9.3$ \\
Affected hemisphere, right/left ( $N)$ & $140 / 53$ \\
Bamford classification, LACI/PACI/TACI (N) & $96 / 52 / 9$ \\
Barthel Index (0-20 points, median (interquartile range)) & $9(5-13)$ \\
\hline LACl lacunar anterior cerebral infarction, PACl partial anterior cerebral \\
infarction, SD standard deviation, TACl total anterior cerebral infarction
\end{tabular}




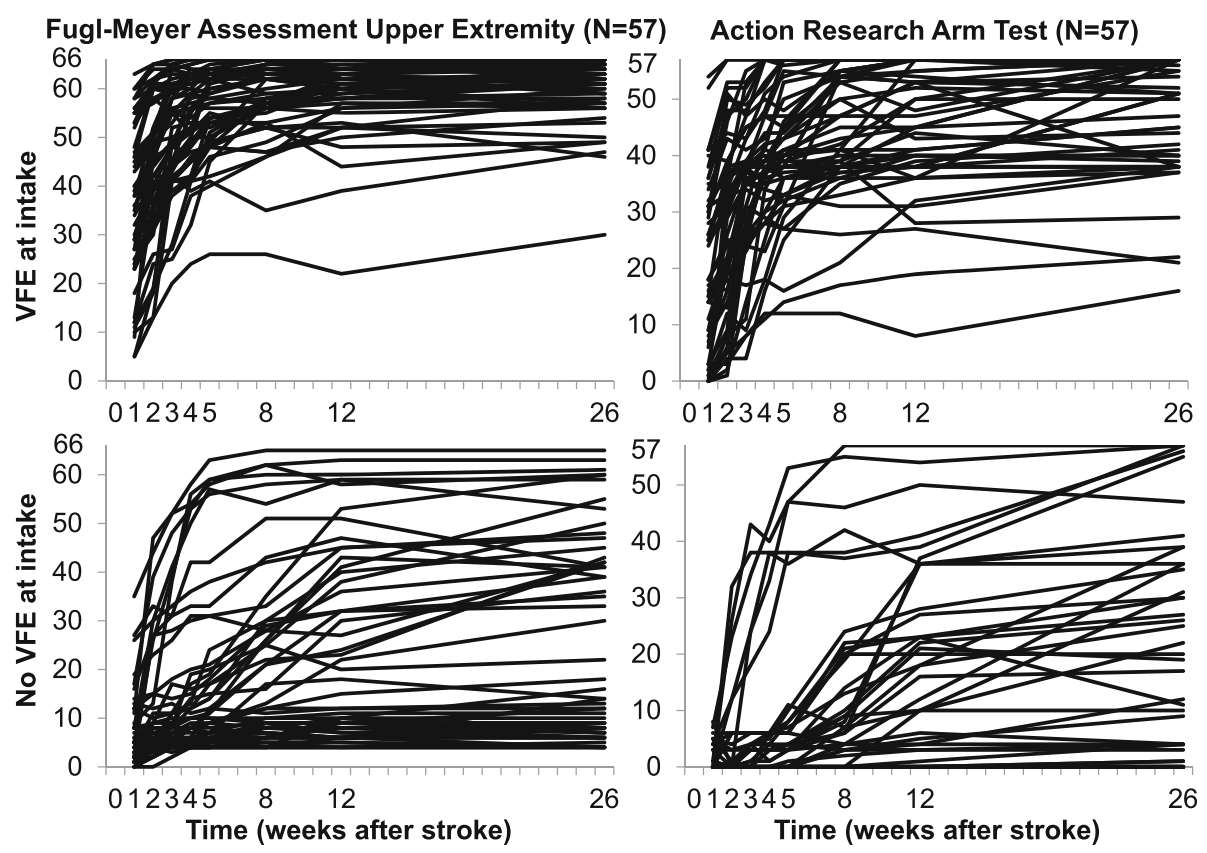

Fig. 1 Individual FMA-UE and ARAT recovery curves for patients with and without Voluntary Finger Extension (VFE). The top two graphs represent the patients with VFE at about 1 week after stroke and the bottom two graphs the patients without VFE. The lefthand two graphs and righthand two graphs represent, respectively, the Upper Extremity motor scores of the Fugl-Meyer Assessment (FMA-UE, score $=0-66,66=$ normal function) and the Action Research Arm Test (ARAT, score $=0-57,57=$ normal capacity)

The required sample sizes for the group of patients without VFE increased when the time interval between stroke onset and randomization was broadened. The largest sample size was found for the broadest time interval, i.e., when randomization was performed between stroke onset and 12 weeks post stroke. For the group of patients with VFE at intake, with the FMA-UE as outcome measure, we found a progressive decrease in sample size when the time interval was broadened. For the ARAT, we observed an increase in the sample sizes when the time interval was broadened from 3 to 5 weeks, after which it remained constant before decreasing to 205 subjects in the fifth time interval. The required sample sizes for the groups of patients with and

Table 2 Results sample size calculation: various time intervals from stroke onset to the moment of randomization $(N=157)$

\begin{tabular}{|c|c|c|c|c|c|}
\hline Time interval & $\begin{array}{l}\text { Group } 1 \\
\text { Mean } \pm \text { SD }\end{array}$ & $\begin{array}{l}\text { Group } 0 \\
\text { Mean } \pm \text { SD }\end{array}$ & SD pooled & Variance & Sample size \\
\hline \multicolumn{6}{|l|}{ FMA-UE } \\
\hline $\mathrm{T}_{0}$ to 1 week & $18.09 \pm 19.78$ & $15.62 \pm 17.06$ & 18.47 & 341 & 275 \\
\hline$T_{0}$ to 3 weeks & $22.19 \pm 21.86$ & $21.23 \pm 20.07$ & 20.98 & 440 & 352 \\
\hline $\mathrm{T}_{0}$ to 5 weeks & $28.89 \pm 23.42$ & $24.27 \pm 21.66$ & 22.56 & 509 & 405 \\
\hline $\mathrm{T}_{0}$ to 8 weeks & $24.54 \pm 22.91$ & $25.80 \pm 22.36$ & 22.64 & 512 & 409 \\
\hline $\mathrm{T}_{0}$ to 12 weeks & $26.18 \pm 24.19$ & $28.69 \pm 22.61$ & 23.42 & 548 & 438 \\
\hline \multicolumn{6}{|l|}{ ARAT } \\
\hline $\mathrm{T}_{0}$ to 1 week & $8.24 \pm 12.82$ & $7.33 \pm 12.89$ & 12.85 & 165 & 178 \\
\hline $\mathrm{T}_{0}$ to 3 weeks & $11.70 \pm 16.76$ & $11.15 \pm 15.96$ & 16.36 & 268 & 288 \\
\hline$T_{0}$ to 5 weeks & $13.58 \pm 17.67$ & $13.77 \pm 17.95$ & 17.81 & 317 & 341 \\
\hline $\mathrm{T}_{0}$ to 8 weeks & $15.65 \pm 19.76$ & $15.14 \pm 19.02$ & 19.39 & 376 & 403 \\
\hline $\mathrm{T}_{0}$ to 12 weeks & $17.95 \pm 21.49$ & $15.46 \pm 18.34$ & 19.98 & 399 & 429 \\
\hline
\end{tabular}

Sample sizes are the total number of patients required, including $10 \%$ to account for dropouts. Individual patients were randomly selected at different time points post stroke onset, where after this assessment was considered as their baseline assessment. Mean and SD are derived from the full dataset, after individual curve fitting. ARAT Action Research Arm Test (score = 0-57, 57 = normal capacity), FMA-UE Upper Extremity motor section of the Fugl-Meyer Assessment (score $=0-66,66=$ normal function), $S D$ standard deviation, $T_{0}$ stroke onset 

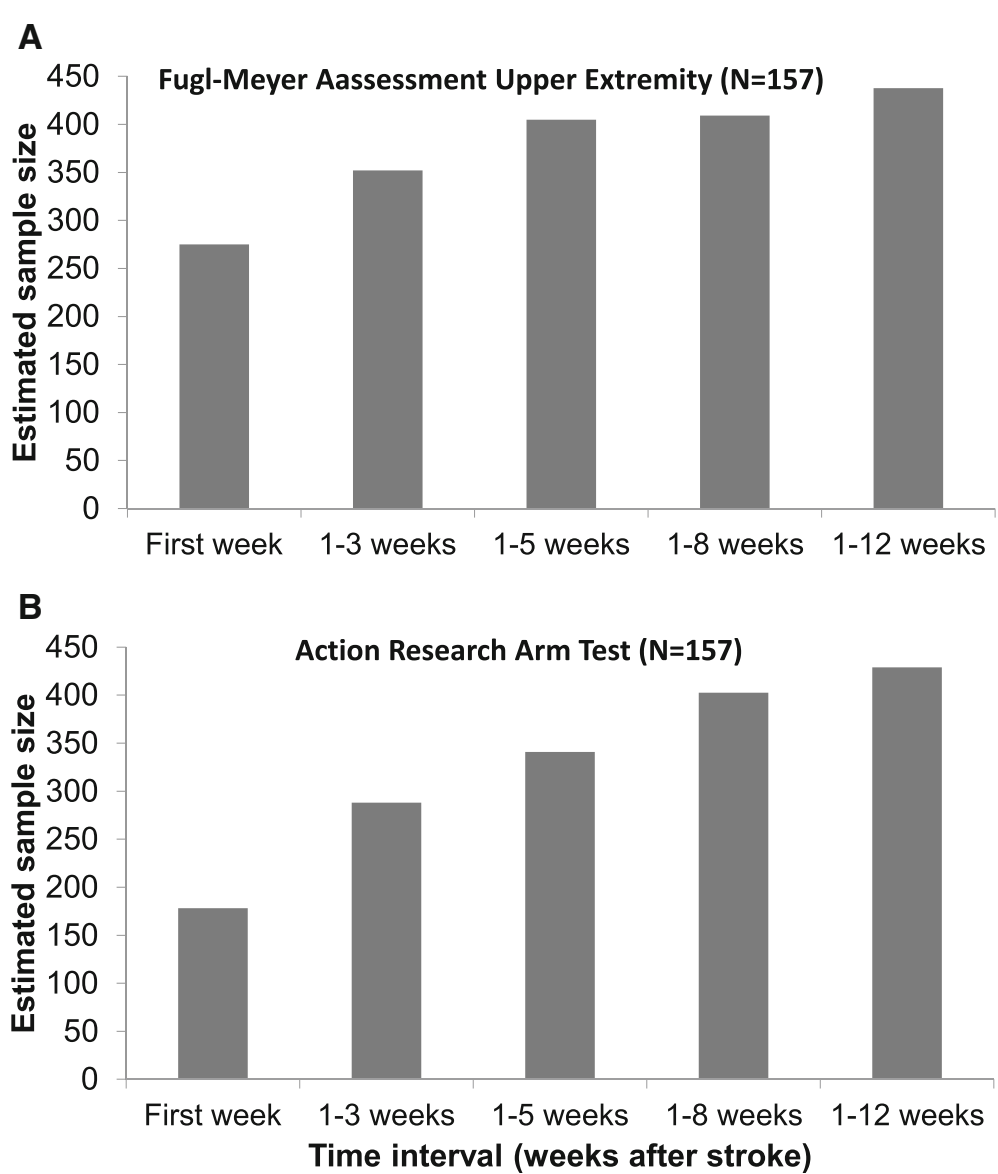

Fig. 2 Impact of the timing of randomization on the sample size. a Upper Extremity motor section of the Fugl-Meyer Assessment, $N=157$. b Action Research Arm Test, $N=157$

without VFE separately remained lower in comparison to the whole group throughout the second to fifth time intervals.

\section{Discussion}

The aim of the present study was to investigate the impact of the timing of randomization and prognostic stratification on the required sample size to reveal significant and clinically important intervention effects on the FMA-UE and ARAT at 26-week follow-up. We used different scenarios for random patient recruitment based on data from a recently published RCT with repeated measuments [16]. We were able to show that the timing of the moment of randomization post stroke, and stratification based on the prognostic determinant VFE, are fundamental for preventing type II errors in neurorehabilitation trials post stroke. This finding is in agreement with the study by Duncan and coworkers [6] who also showed that the length of time from stroke onset (baseline, 5-day or 30-day status) and the severity of motor impairment measured with the FMA motor score (mild to severe) influences the chance of showing $50 \%$ improvement in the residual motor deficit. In the present study, randomization of patients at arbitrary time points post stroke (i.e., wide time intervals) showed a tremendous increase in the required sample size. The results underpin the importance of carefully designing future RCTs to increase the chance of finding differential intervention effects. At present, upper limb trials with 2 experimental arms, assuming $80 \%$ statistical power with randomization at a fixed moment in the first 3 months after stroke, are lacking in the scientific literature.

Lack of prognostic stratification would give an incomplete representation of the changes in underlying subgroups. Interestingly, the timing of the moment of randomization and stratification of subjects in upper limb trials are not independent phenomena for estimation of the number of subjects required for sufficiently powered rehabilitation trials. In the stratified group of patients with VFE within the first week, we observed a slight, overall decrease in the heterogeneity between outcome scores of patients when the time interval between stroke onset and randomization was increased. After 3 months, the group of mild to moderately 
Table 3 Results sample size calculation: stratification based on presence or absence of voluntary finger extension

\begin{tabular}{|c|c|c|c|c|c|c|c|c|c|c|c|c|c|c|c|}
\hline \multirow[b]{2}{*}{ Time interval } & \multicolumn{5}{|c|}{ All patients $(N=114)$} & \multicolumn{5}{|c|}{ Voluntary Finger Extension $(N=57)$} & \multicolumn{5}{|c|}{ No Voluntary Finger Extension $(N=57)$} \\
\hline & $\begin{array}{l}\text { Group } 1 \\
\text { Mean } \pm \text { SD }\end{array}$ & $\begin{array}{l}\text { Group } 0 \\
\text { Mean } \pm \text { SD }\end{array}$ & $\begin{array}{l}\text { SD } \\
\text { pooled }\end{array}$ & Variance & $\begin{array}{l}\text { Sample } \\
\text { size }\end{array}$ & $\begin{array}{l}\text { Group } 1 \\
\text { Mean } \pm \text { SD }\end{array}$ & $\begin{array}{l}\text { Group } 0 \\
\text { Mean } \pm \text { SD }\end{array}$ & $\begin{array}{l}\text { SD } \\
\text { pooled }\end{array}$ & Variance & $\begin{array}{l}\text { Sample } \\
\text { size }\end{array}$ & $\begin{array}{l}\text { Group } 1 \\
\text { Mean } \pm \text { SD }\end{array}$ & $\begin{array}{l}\text { Group } 0 \\
\text { Mean } \pm \text { SD }\end{array}$ & $\begin{array}{l}\text { SD } \\
\text { pooled }\end{array}$ & Variance & Sample size \\
\hline \multicolumn{16}{|l|}{ FMA-UE } \\
\hline To to 1 week & $23.17 \pm 20.80$ & $19.73 \pm 18.36$ & 19.61 & 385 & 308 & $39.31 \pm 16.59$ & $33.68 \pm 15.74$ & 16.17 & 262 & 211 & $7.03 \pm 8.11$ & $5.79 \pm 5.92$ & 7.10 & 50 & 44 \\
\hline$T_{0}$ to 3 weeks & $29.22 \pm 23.10$ & $26.21 \pm 20.61$ & 21.89 & 476 & 385 & $45.55 \pm 15.19$ & $42.50 \pm 15.62$ & 15.40 & 237 & 191 & $9.62 \pm 9.62$ & $7.86 \pm 7.84$ & 8.77 & 77 & 64 \\
\hline $\mathrm{T}_{0}$ to 5 weeks & $32.05 \pm 23.61$ & $30.57 \pm 21.73$ & 22.69 & 515 & 411 & $50.24 \pm 14.88$ & $48.46 \pm 11.85$ & 13.45 & 181 & 147 & $9.97 \pm 11.32$ & $13.21 \pm 13.39$ & 12.40 & 154 & 125 \\
\hline $\mathrm{T}_{0}$ to 8 weeks & $30.83 \pm 24.00$ & $33.36 \pm 22.76$ & 23.39 & 547 & 438 & $49.41 \pm 13.80$ & $49.75 \pm 12.90$ & 13.36 & 178 & 145 & $12.38 \pm 12.98$ & $11.64 \pm 13.79$ & 13.39 & 179 & 145 \\
\hline $\mathrm{T}_{0}$ to 12 weeks & $34.33 \pm 25.09$ & $31.09 \pm 22.44$ & 23.80 & 567 & 453 & $51.03 \pm 14.22$ & $51.29 \pm 12.14$ & 13.22 & 175 & 143 & $16.24 \pm 17.04$ & $16.04 \pm 16.15$ & 16.60 & 276 & 222 \\
\hline \multicolumn{16}{|l|}{ ARAT } \\
\hline$T_{0}$ to 1 week & $10.91 \pm 13.98$ & $9.91 \pm 14.39$ & 14.19 & 201 & 218 & $20.62 \pm 14.06$ & $19.21 \pm 15.48$ & 14.79 & 219 & 235 & $1.21 \pm 2.29$ & $0.61 \pm 1.69$ & 2.01 & 4 & 9 \\
\hline$T_{0}$ to 3 weeks & $16.16 \pm 17.60$ & $14.98 \pm 17.14$ & 17.37 & 302 & 323 & $29.07 \pm 16.06$ & $27.54 \pm 15.62$ & 15.84 & 251 & 271 & $2.48 \pm 6.10$ & $0.82 \pm 1.98$ & 4.54 & 21 & 24 \\
\hline $\mathrm{T}_{0}$ to 5 weeks & $18.76 \pm 19.72$ & $18.21 \pm 19.08$ & 19.40 & 376 & 403 & $34.35 \pm 16.45$ & $28.36 \pm 15.13$ & 15.80 & 250 & 268 & $2.55 \pm 6.75$ & $3.61 \pm 7.89$ & 7.34 & 54 & 62 \\
\hline $\mathrm{T}_{0}$ to 8 weeks & $22.22 \pm 20.85$ & $17.20 \pm 19.01$ & 19.95 & 398 & 425 & $34.93 \pm 16.57$ & $32.00 \pm 15.18$ & 15.89 & 253 & 271 & $5.07 \pm 10.48$ & $3.71 \pm 6.77$ & 8.82 & 78 & 86 \\
\hline $\mathrm{T}_{0}$ to 12 weeks & $22.33 \pm 22.04$ & $21.48 \pm 19.80$ & 20.95 & 439 & 471 & $38.17 \pm 13.31$ & $35.79 \pm 14.12$ & 13.72 & 188 & 205 & $4.03 \pm 9.14$ & $5.61 \pm 12.39$ & 10.89 & 119 & 130 \\
\hline
\end{tabular}

Sample sizes are the total number of patients required, including $10 \%$ to account for dropouts. Individual patients were randomly selected at different time points post stroke onset, where after this assessment was considered as their baseline assessment. Mean and SD are derived from the full dataset, after individual curve fitting. ARAT Action Research Arm Test (score $=0-57,57=$ normal capacity), FMA-UE Upper Extremity motor section of the Fugl-Meyer Assessment (score $=0-66,66=$ normal function), $S D=$ standard deviation, $T_{0}$ stroke onset 


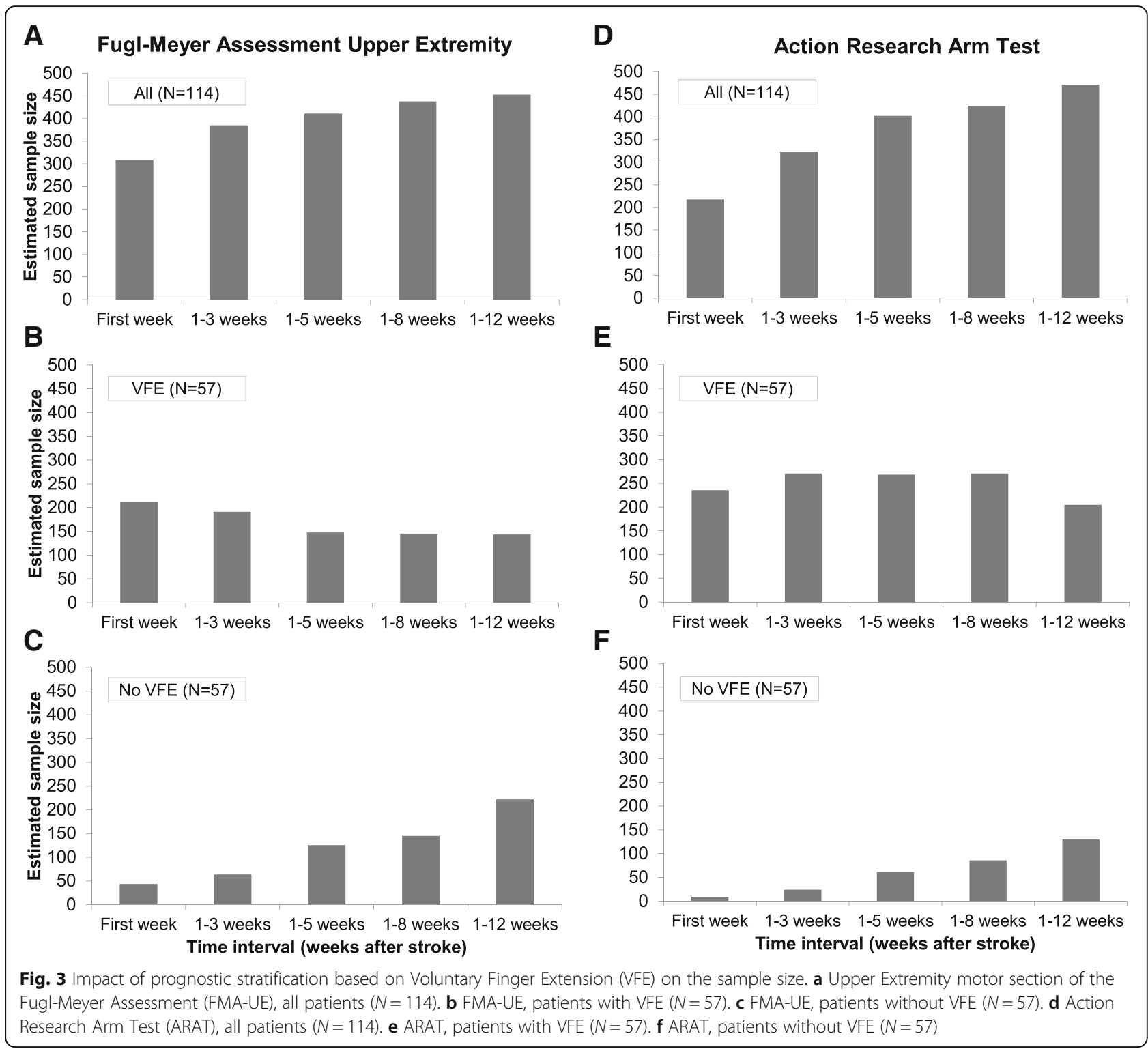

impaired patients was more homogenous and, as a consequence, significant smaller sample sizes were required to find clinical meaningful effects of $10 \%$ on the FMA-UE or ARAT.

The heterogeneity between outcome scores of patients in the stratified group of patients with severe motor impairments (i.e., no VFE) was low when randomization occurred in the first few weeks after stroke onset. Thereafter, the heterogeneity between patients increased substantially when the time interval for randomization was extended to 8 or 12 weeks post stroke. As previous studies showed, severely impaired patients will most likely reach their plateau in motor recovery later in time in comparison to mild to moderately impaired patients $[6,7]$. The majority of these severely impaired patients will only show minimal improvement in upper limb function and there are still no evidence-based interventions for this specific group of patients $[1,11,16]$. However, a portion of the severely impaired patients in the current dataset displayed more recovery of upper limb function than predicted (i.e., mainly "false negatives"), which markedly increased the heterogeneity in outcome scores between patients when the time intervals for randomization were broadened [12].

Recent prospective cohort studies have suggested that the amount of spontaneous neurobiological recovery is highly predictable within the first $72 \mathrm{~h}$ after stroke onset, and that the majority of patients will recover to a level of about 70 to $80 \%$ of their maximum possible improvement based on their initial impairment [14, 27-30]. At present, we can only assign patients retrospectively and discriminate between "fitters" and "non-fitters" in terms 
of expected spontaneous neurobiological recovery. There is a need for prospective stratification of patients according to their potential neurobiological recovery determined early after stroke [14]. Therefore, prognostic biomarkers are needed to identify patients who will and will not show the expected spontaneous biological recovery next to robust clinical markers such as VFE. In addition, we are of the opinion that the above recommendations with respect to the timing of randomization procedures and applying stratification in designing upper limb trials are not unique for upper limb trials. In particular, acknowledging that outcomes of the lower paretic limb parallel those of the upper limb [6,7] and that the prognosis of meaningful outcomes, such as walking ability, is strongly dependent on initial sitting balance and lower limb strength [31]. In addition, there is growing evidence that this maximum proportional recovery rule of spontaneous neurobiological recovery is not restricted to motor recovery alone, but also applies to cognitive impairments such as visuospatial neglect and aphasia [28]. This latter finding suggests that the current recommendations for designing trials are probably generalizable to other modalities affected after stroke.

The following points should be taken into account when interpreting the results. First, generalizability of the current results may be limited because the estimates were derived from a single study of a relatively small population of patients with first-ever ischemic hemispheric stroke. Despite this small population, the heterogeneity between patients in the current study is representative for other stroke populations $[6,12,32]$. Second, we only included two important factors for designing an RCT in the present study. Other important factors that should be taken into account are, for example: the biological rationale behind the research protocol including selection of research interventions and dose of therapy, and selecting the appropriate outcome measures [33, 34]. Third, the prognostic stratification that was used in the original RCT was based on the first assessment at approximately 1 week after stroke. We used the SDs determined for different time intervals as a representation for the SDs at 26-week followup. We did not, however, account for possible changes in prognosis for upper limb capacity over time (i.e., return of VFE). As there were a number of patients in the group without VFE at intake who showed more recovery than expected based on their prognosis early after stroke, the SDs for the first few time intervals in the group of patients without VFE may not be completely representative for the $\mathrm{SD}$ at 26-week follow-up. Fourth, the estimated sample sizes in the first few time intervals with the ARAT were very small (Tables 2 and 3). The estimated sample sizes were even smaller in solely the group of patients without VFE. These results point out the considerable impact of prognostic stratification and time between stroke onset and randomization in RCTs. However, we do not recommend that researchers design RCTs with these very small numbers of participants as they will be prone to error. Fifth, for modeling purposes we assumed a normally distributed outcome and performed a standard $t$ test sample size calculation, commonly used in stroke trials. If the distribution of the outcome was not normally distributed, a rank-based test may have been more appropriate. Sixth, a sample size estimation based on a standard $t$ test may overestimate the sample size compared to a stratified version of the test or a regression model. Higher power may be attained when stratification is included in the analysis stage.

\section{Conclusion}

The timing of the moment of randomization post stroke, and stratification based on the prognostic determinant VFE, are fundamental for designing upper limb trials early post stroke. To increase the chance of finding differential intervention effects, future RCTs should randomize patients at fixed moments after stroke and stratify patients according to their potential neurobiological recovery.

\section{Abbreviations \\ ARAT: Action Research Arm Test; EMG-NMS: EMG-triggered Neuro-muscular Stimulation; FMA-UE: Fugl-Meyer Assessment Upper Extremity; MCID: Minimal Clinically Important Difference; mCIMT: Modified Constraint-induced Movement Therapy; NIHSS: National Institutes of Health Stroke Scale; RCT: Randomized controlled trial; VFE: Voluntary Finger Extension}

\section{Acknowledgements}

The authors thank the EXPLICIT-stroke trial physicians, therapist and nurses at the stroke units of the participating university centers and local hospitals and in the associated rehabilitation centers and nursing homes, and the patients who participated in the study.

\section{Funding}

The research leading to these results has received funding from the EXPLICIT-stroke trial grant from the Dutch Organization for Health Research and Development (ZonMw grant no. 89000001), supported by the European Research Council under the European Union's Seventh Framework Program (FP/2007-2013)/ERC Grant Agreement no. 291339-4D-EEG. The funding organizations had no role in the design or conduct of the study; the collection, management, analysis or interpretation of the data; or the preparation, review or approval of the manuscript.

\section{Availability of data and materials}

The dataset supporting the conclusions of this article is available upon request. Requests for data access can be submitted to the corresponding author who will evaluate the request with the funding organization (ZonMw grant no. 98000001) as well as the EXPLICIT-stroke trial consortium (Ethics Committee Leiden University Medical Center; no. P08.035).

\section{Authors' contributions}

CW conceived and designed the study, and was responsible for data analysis, drafting the manuscript and the figures. MH was responsible for data analysis and drafting the manuscript. EW and GK conceived and designed the study, and were responsible for drafting the manuscript. All authors read and approved the final manuscript.

\section{Competing interests}

The authors declare that they have no competing interests.

Consent for publication

Not applicable. 


\section{Ethics approval and consent to participate}

The EXPLICIT-stroke trial was approved by the Medical Ethical Committee of the Leiden University Medical Center (P08.035; CCMO no. NL21396.058.08), funded by the Netherlands Organization for Health Research and Development (ZonMw grant no. 890000001). Written informed consent was obtained from all patients.

\section{Author details}

'Department of Rehabilitation Medicine, VU University Medical Center, MOVE Research Institute Amsterdam, Amsterdam, The Netherlands. ${ }^{2}$ Neuroscience Campus Amsterdam, Vrije Universiteit, Amsterdam, The Netherlands. ${ }^{3}$ Department of Epidemiology and Biostatistics, VU University Medical Center Amsterdam, Amsterdam, The Netherlands. ${ }^{4}$ Department of Methodology and Applied Biostatistics, Faculty of Earth and Life Sciences, Vrije Universiteit, Amsterdam, The Netherlands. ${ }^{5}$ Amsterdam Rehabilitation Research Center, Reade, Amsterdam, The Netherlands. ${ }^{6}$ Department of Physical Therapy and Human Movement Sciences, Northwestern University, Chicago, IL, USA.

\section{Received: 29 June 2016 Accepted: 8 September 2016}

\section{Published online: 26 September 2016}

\section{References}

1. Veerbeek JM, van Wegen, van Peppen $R$, van der Wees PJ, Hendriks E, Rietberg $\mathrm{M}$, et al. What is the evidence for physical therapy poststroke? A systematic review and meta-analysis. PLoS One. 2014;9:e87987.

2. Pollock A, Farmer SE, Brady MC, Langhorne P, Mead GE, Mehrholz J, et al. Interventions for improving upper limb function after stroke. Cochrane Database Syst Rev. 2014;11, CD010820.

3. Murphy $\mathrm{TH}$, Corbett D. Plasticity during stroke recovery: from synapse to behaviour. Nat Rev Neurosci. 2009;10:861-72.

4. Krakauer JW, Carmichael ST, Corbett D, Wittenberg GF. Getting neurorehabilitation right: what can be learned from animal models? Neurorehabil Neural Repair. 2012;26:923-31.

5. Carmichael ST, Krakauer JW. The promise of neuro-recovery after stroke: introduction. Stroke. 2013:44:S103.

6. Duncan PW, Goldstein LB, Matchar D, Divine GW, Feussner J. Measurement of motor recovery after stroke. Outcome assessment and sample size requirements. Stroke. 1992;23:1084-9.

7. Kwakkel G, Kollen B, Twisk J. Impact of time on improvement of outcome after stroke. Stroke. 2006;37:2348-53.

8. Cramer SC. Repairing the human brain after stroke: I. Mechanisms of spontaneous recovery. Ann Neurol. 2008;63:272-87.

9. Nijboer TC, Kollen BJ, Kwakkel G. The impact of recovery of visuo-spatial neglect on motor recovery of the upper paretic limb after stroke. PLoS One. 2014;9, e100584

10. Langhorne P, Bernhardt J, Kwakkel G. Stroke rehabilitation. Lancet. 2011;377:1693-702.

11. Langhorne $\mathrm{P}$, Coupar F, Pollock A. Motor recovery after stroke: a systematic review. Lancet Neurol. 2009;8:741-54.

12. Nijland $\mathrm{RH}$, van Wegen EE, Harmeling-van der Wel BC, Kwakkel G. Presence of finger extension and shoulder abduction within 72 hours after stroke predicts functional recovery: early prediction of functional outcome after stroke: the EPOS cohort study. Stroke. 2010;41:745-50.

13. Stinear C. Prediction of recovery of motor function after stroke. Lancet Neurol. 2010:9:1228-32.

14. Byblow WD, Stinear CM, Barber PA, Petoe MA, Ackerley SJ. Proportional recovery after stroke depends on corticomotor integrity. Ann Neurol. 2015;78:848-59.

15. Sunderland A, Tinson DJ, Bradley EL, Fletcher D, Langton HR, Wade DT. Enhanced physical therapy improves recovery of arm function after stroke. A randomised controlled trial. J Neurol Neurosurg Psychiatry. 1992;55:530-5.

16. Kwakkel G, Winters C, van Wegen EE, Nijland RH, van Kuijk AA, Visser-Meily $A$, et al. Effects of unilateral upper limb training in two distinct prognostic groups early after stroke: the EXPLICIT-stroke randomized clinical trial. Neurorehabil Neural Repair. 2016. [Epub ahead of print 7 Jan 2016].

17. Kwakkel G, Veerbeek JM, van Wegen EE, Wolf SL. Constraint-induced movement therapy after stroke. Lancet Neurol. 2015;14:224-34.

18. Jolkkonen J, Kwakkel G. Translational hurdles in stroke recovery studies. Transl Stroke Res. 2016. [Epub ahead of print 22 Mar 2016].

19. Kwakkel G, Meskers CG, van Wegen EE, Lankhorst GJ, Geurts AC, van Kuijk AA et al. Impact of early applied upper limb stimulation: the EXPLICIT-stroke programme design. BMC Neurol. 2008:8:49.
20. Lyle RC. A performance test for assessment of upper limb function in physical rehabilitation treatment and research. Int J Rehabil Res. 1981:4:483-92.

21. Yozbatiran N, Der-Yeghiaian L, Cramer SC. A standardized approach to performing the action research arm test. Neurorehabil Neural Repair. 2008;22:78-90.

22. Van der Lee JH, Wagenaar RC, Lankhorst GJ, Vogelaar TW, Deville WL, Bouter LM. Forced use of the upper extremity in chronic stroke patients: results from a single-blind randomized clinical trial. Stroke. 1999;30:2369-75.

23. Fugl-Meyer AR, Jaasko L, Leyman I, Olsson S, Steglind S. The post-stroke hemiplegic patient. 1. a method for evaluation of physical performance. Scand J Rehabil Med. 1975;7:13-31

24. Sanford J, Moreland J, Swanson LR, Stratford PW, Gowland C. Reliability of the Fugl-Meyer assessment for testing motor performance in patients following stroke. Phys Ther. 1993;73:447-54.

25. Van der Lee JH, Beckerman H, Lankhorst GJ, Bouter LM. The responsiveness of the Action Research Arm test and the Fugl-Meyer Assessment scale in chronic stroke patients. J Rehabil Med. 2001;33:110-3.

26. Gladstone DJ, Danells CJ, Black SE. The Fugl-Meyer assessment of motor recovery after stroke: a critical review of its measurement properties. Neurorehabil Neural Repair. 2002;16:232-40.

27. Prabhakaran S, Zarahn E, Riley C, Speizer A, Chong JY, Lazar RM, et al. Inter-individual variability in the capacity for motor recovery after ischemic stroke. Neurorehabil Neural Repair. 2008:22:64-71.

28. Lazar RM, Minzer B, Antoniello D, Festa JR, Krakauer JW, Marshall RS. Improvement in aphasia scores after stroke is well predicted by initial severity. Stroke. 2010;41:1485-8.

29. Winters C, van Wegen EE, Daffertshofer A, Kwakkel G. Generalizability of the Proportional Recovery Model for the upper extremity after an ischemic stroke. Neurorehabil Neural Repair. 2015;29:614-22.

30. Krakauer JW, Marshall RS. The proportional recovery rule for stroke revisited. Ann Neurol. 2015;78:845-7.

31. Veerbeek JM, van Wegen EE, Harmeling-van der Wel BC, Kwakkel G. Is accurate prediction of gait in nonambulatory stroke patients possible within 72 hours poststroke? The EPOS study. Neurorehabil Neural Repair. 2011;25:268-74

32. Kwakkel G, Kollen BJ, van der Grond J, Prevo AJ. Probability of regaining dexterity in the flaccid upper limb: impact of severity of paresis and time since onset in acute stroke. Stroke. 2003:34:2181-6.

33. Ali M, English C, Bernhardt J, Sunnerhagen KS, Brady M. More outcomes than trials: a call for consistent data collection across stroke rehabilitation trials. Int J Stroke 2013:8:18-24.

34. Duncan PW. Outcome measures in stroke rehabilitation. Handb Clin Neurol. 2013:110:105-11.

\section{Submit your next manuscript to BioMed Central and we will help you at every step:}

- We accept pre-submission inquiries

- Our selector tool helps you to find the most relevant journal

- We provide round the clock customer support

- Convenient online submission

- Thorough peer review

- Inclusion in PubMed and all major indexing services

- Maximum visibility for your research

Submit your manuscript at www.biomedcentral.com/submit
) Biomed Central 